\title{
Motion Artifacts, Motion Compensation, and Magnetic Resonance Angiography
}

\section{INTRODUCTION}

$\mathbf{M}$

otion artifacts can cause both blurring and ghosting of an image. It is thus important

to understand the source of these artifacts in order to be able to avoid or eliminate them. UNIT B7.1 discusses the phase induced by motion and how it leads to these artifacts. Effects caused by motion in the read and phase-encoding direction are discussed, with the latter shown to be the culprit producing the majority of the artifacts. If this extra phase dependence on motion or flow can be eliminated, then the artifacts will be removed. This is best accomplished by redesigning the gradients in the sequence so that the motion-induced phase is zero at the echo time. Such a sequence is referred to as a velocity-compensated (gradient-moment-nulling) sequence. This is the subject of UNIT B7.2. Blood flow in vessels suffers from the above mentioned artifacts unless these velocity-compensation techniques are used. When these special motion compensation methods are successful, the blood vessels become very bright and are easily distinguished from the surrounding tissue. Imaging vessels using MRI is referred to as magnetic resonance angiography (MRA). In UNIT B7.3, the inflow effects (i.e., how the speed of blood flow affects the signal intensity of the blood in the images) is discussed. In addition, how the use of a contrast agent can be used to reduce $T_{1}$ and thus further enhance the blood's signal for a short $T_{E}, T_{1}$-weighted sequence is also treated in this unit.

Yu-Chung Norman Cheng and E. Mark Haacke 